\title{
Multilevel Barriers to HIV PrEP Uptake and Adherence Among Black and Hispanic/Latinx Transgender Women in Southern California
}

\author{
Adedotun Ogunbajo ${ }^{1}$ [ - Erik D. Storholm ${ }^{2,3,4}$ - Allison J. Ober ${ }^{3,4}$ - Laura M. Bogart ${ }^{3,4}$ - Cathy J. Reback ${ }^{4,5}$. \\ Risa Flynn ${ }^{6} \cdot$ Phoebe Lyman $^{6} \cdot$ Sheldon Morris ${ }^{7}$
}

Accepted: 8 January 2021 / Published online: 29 January 2021

(c) The Author(s), under exclusive licence to Springer Science+Business Media, LLC part of Springer Nature 2021

\begin{abstract}
Black and Hispanic/Latinx transgender women in the United States (U.S.) are disproportionately affected by HIV. Preexposure prophylaxis (PrEP) reduces risk of HIV infection but PrEP uptake remains low among Black and Hispanic/Latinx transgender women. Between July 2018 and August 2019, we conducted individual interviews with 30 Black and Hispanic/ Latinx transgender women who were prescribed PrEP through a PrEP demonstration project and 10 healthcare providers who provide PrEP services to transgender women in Los Angeles and San Diego, California. The interviews assessed general attitudes, experiences, and beliefs about PrEP as well as individual-, interpersonal-, community-, and structural-level barriers to PrEP uptake and adherence. PrEP adherence was assessed by collecting quantitative intracellular tenofovir-diphosphate (TFV-DP) levels in retrospect on batched, banked dried blood spot (DBS) samples. We utilized qualitative content analysis to identify themes from the interviews. Findings indicated the presence of individual-level barriers including cost concerns, mental health issues, substance use, and concerns about PrEP side effects including hormone interaction. Interpersonal-level barriers included the influence of intimate/romantic partners and the impact of patient-provider communication. Communitylevel barriers consisted of experiencing stigma and negative community opinions about PrEP use as well as having negative experiences in healthcare settings. Structural-level barriers included unreliable transportation, employment, and housing insecurity. Interventions aiming to increase PrEP uptake and adherence among Black and Hispanic/Latinx transgender women in the U.S. should employ a multilevel approach to addressing the needs of transgender women, especially the structural barriers that have greatly limited the use of PrEP.
\end{abstract}

Keywords Transgender women $\cdot$ PrEP adherence $\cdot$ Black $\cdot$ Hispanic/Latinx $\cdot$ HIV prevention

Adedotun Ogunbajo

dotunogunbajo@gmail.com

1 Department of Epidemiology, Harvard T.H. Chan School of Public Health, Boston, MA, USA

2 School of Public Health, San Diego State University, San Diego, CA, USA

3 RAND Corporation, Santa Monica, CA, USA

4 Department of Family Medicine, Center for HIV Identification, Prevention and Treatment Services, University of California Los Angeles, Los Angeles, CA, USA

5 Friends Research Institute, Los Angeles, CA, USA

6 Los Angeles LGBT Center, Los Angeles, CA, USA

7 AntiViral Research Center, San Diego School of Medicine, University of California, San Diego, CA, USA 


\section{Resumen}

Las mujeres transexuales negras e hispanas/latinx en los Estados Unidos (EE. UU.) se ven afectadas de manera desproporcionada por el VIH. La profilaxis previa a la exposición (PrEP) reduce el riesgo de infección por VIH, pero la aceptación sigue siendo baja entre las mujeres transgénero negras e hispanas/latinx. Entre julio de 2018 y agosto de 2019, realizamos entrevistas individuales con 30 mujeres transgénero negras e hispanas/latinx a las que se les recetó PrEP a través de un proyecto de demostración de PrEP y a 10 proveedores de atención médica que brindan servicios de PrEP a mujeres transgénero en Los Ángeles y San Diego, California. Las entrevistas evaluaron las actitudes, experiencias y creencias generales sobre PrEP, así como las barreras a nivel individual, interpersonal, comunitario y estructural para la aceptación y la adherencia. La adherencia a PrEP se evaluó mediante la recolección de niveles intracelulares cuantitativos de difosfato de tenofovir (TFV-DP) en retrospectiva en muestras de manchas de sangre seca (DBS) acumuladas y almacenadas. Utilizamos análisis de contenido cualitativo para identificar temas de las entrevistas. Los hallazgos indicaron la presencia de barreras a nivel individual, incluidas preocupaciones de costos, problemas de salud mental, uso de sustancias y preocupaciones sobre los efectos secundarios de PrEP, incluida la interacción hormonal. Las barreras a nivel interpersonal incluyeron la influencia de las parejas íntimas/románticas y el impacto de la comunicación entre el paciente y el proveedor. Las barreras a nivel de la comunidad consistieron en experimentar estigma y opiniones negativas de la comunidad sobre el uso de PrEP, así como tener experiencias negativas en entornos de atención médica. Las barreras a nivel estructural incluían transporte poco confiable, así como inseguridad en el empleo y la vivienda. Las intervenciones destinadas a aumentar la aceptación y el cumplimiento de PrEP entre las mujeres transgénero negras e hispanas/latinx en los EE. UU. deben emplear un enfoque multinivel para abordar las necesidades de las mujeres transgénero, especialmente las barreras estructurales que han limitado en gran medida el uso de PrEP.

\section{Introduction}

Transgender women-those who were assigned male at birth and who identity as female-are disproportionately affected by the HIV epidemic in the United States (U.S.) [1]. Between 2009 and 2014, an estimated 1974 transgender women were diagnosed with HIV in the U.S., representing $84 \%$ of transgender people diagnosed [1]. A recently published systematic review and metaanalysis on transgender populations in the U.S estimated that $14.1 \%$ of transgender women in the U.S. are living with HIV [2]. Racial and ethnic minority transgender women bear a higher burden of HIV compared to White transgender women. In the U.S., an estimated $44 \%$ of Black transgender women and $26 \%$ of Latinx/Hispanic transgender women are living with HIV compared to $7 \%$ of White transgender women [2]. Major drivers of the substantial disparity in HIV rates among Black, Hispanic/ Latinx, and White transgender women include experiences of discrimination and stigma, limited access to healthcare services, lack of education and employment opportunities, and unstable housing [3].

Pre-exposure prophylaxis (PrEP) is a biomedical intervention that reduces the risk of HIV transmission, when taken as prescribed [4-6]. While research has demonstrated high willingness to take PrEP among transgender women [7-10], uptake remains low at about $17 \%$ among Black and Latinx transgender women who would benefit from a biomedical HIV prevention strategy [11, 12]. Given the high burden of HIV among Black and Hispanic/Latinx transgender women, there is a need to better understand the specific barriers to PrEP uptake and adherence affecting these communities.

Previous work has suggested that barriers to PrEP uptake among transgender women fall along multiple socio-ecological levels [13, 14]. Individual-level barriers may include low PrEP awareness and knowledge, past experiences of violence, uncertainty about health insurance coverage and associated health costs, concerns about side effects, possible hormone interactions, past negative healthcare experiences, and self-perception of being at low risk for HIV infection [13-15]. Some examples of interpersonal-level barriers include lack of support of cisgender male partners for PrEP, and medical mistrust due to experiences of transphobia [14, 15]. Structural-level barriers include lack of trans-inclusive PrEP marketing, lack of integration of PrEP services into feminizing hormone therapy (FHT) and gender transition care, and social marginalization (lack of adequate employment and stable housing) [14, 16, 17].

Experiences of social marginalization on the basis of gender, class, race, sexual identity, poverty, and the intersection of these identities and experiences may contribute to increased vulnerability to HIV infection among Black and Hispanic/Latinx transgender women [18]. Although several studies have investigated barriers and facilitators to PrEP uptake among transgender women, there is a dearth of research on multilevel barriers to PrEP uptake and adherence specifically among Black and Hispanic/Latinx transgender women. 


\section{Conceptual Framework Guiding the Current Study}

For the current study, we utilized a socioecological framework [19] to inform the development of the interview protocol and thematic content analysis. The socioecological framework posits that the individual exists within larger systems (microsystem, mesosystem, exosystem, and macrosystem) and these higher level systems directly influence individual behavior. For the purposes of this study, we developed a research protocol that would assess for any possible barriers to PrEP uptake or adherence based on the following levels of the socioecological framework: individual, interpersonal, community, and structural. Individual-level barriers were defined as experiences that significantly affect individual attitudes and/or behaviors (e.g., mental health problems or substance use patterns). Interpersonal-level barriers were defined as factors stemming from interactions between the participant and other significant individuals in their lives (e.g., friends, family members, romantic and/or sexual partners, or healthcare providers). Community-level barriers were defined as barriers that may stem from larger social networks and communities (e.g., mistrust of medical providers or of medicine). Structural-level barriers were defined as factors at the systems level that greatly influence individuals' social capital and access to resources (e.g., transportation or employment).

Previous studies have applied the socioecological framework to understanding attitudes and beliefs about PrEP among sexual minority communities [20-22]. Adopting this framework to our study allowed us to broaden our understanding of the lived experiences of Black and Hispanic/ Latinx transgender women and how the culmination of multilevel experiences influence individual-level behavior. The current study aimed to gain understanding of the multilevel barriers to PrEP uptake and adherence by interviewing a sample of Black and Hispanic/Latinx transgender women in Southern California (Los Angeles and San Diego) who had recently been enrolled in a PrEP demonstration project as well as a sample of healthcare providers (HCP) that provide PrEP-related health services to Black and Hispanic/Latinx transgender women.

\section{Methods}

\section{Parent Study}

The Black and Hispanic/Latinx transgender women enrolled in the current study were recruited from a
Southern California-based PrEP demonstration project among transgender people conducted by the California Collaborative Treatment Group (CCTG) (NCT3086200). The study, "CCTG 603: Randomized Controlled Trial of TAB Plus Motivational Interviewing for PrEP Adherence in Transgender Individuals (iM-PrEPT)," was a randomized, open, two parallel arms, clinical trial of text messaging alone or text messaging plus brief motivational interviewing to improve PrEP adherence in transgender individuals who are at high risk for HIV infection. Participants were enrolled between July 2017 and September 2019. The trial was implemented at: (1) three academic clinics in the California Collaborative Treatment Group network (University of California, Los Angeles, University of Southern California, and University of California, San Diego), and (2) two community sites that provide transgender healthcare services (Los Angeles LGBT Center and the Family Health Centers of San Diego). As part of participation in the demonstration project, all participants were provided PrEP free-of-charge.

Inclusion criteria for the PrEP demonstration project included: (1) self-identifying as transgender or non-binary; (2) confirmed HIV-negative status at baseline; (3) selfidentifying as being at risk for HIV infection based on self-reported condomless sex with a cisgender male partner in the previous 6 months; and, (4) reporting willingness to take PrEP as part of an HIV prevention study. All participants were confirmed to be HIV-uninfected through HIV antibody testing and to have no contraindication to the study drug tenofovir/emtricitabine (TDF/FTC). Participants were followed for 48 weeks; quarterly visits included routine HIV testing and counseling, STI testing, adherence counseling, and medical monitoring.

\section{Current Study}

The goal of this qualitative sub-study was to gain a deeper understanding of the barriers to adherence experienced among Black and Hispanic/Latinx transgender women and non-binary individuals determined to be at high risk for HIV who had been provided TDF/FTC for PrEP as part of a larger demonstration project. Specifically, we aimed to gain insight into the barriers to adherence among a sample of transgender women who exhibited either protective or non-protective levels of TDF/FTC in their participant blood samples collected 12 weeks into the study. Therefore, additional inclusion criteria for the sub-study included identifying specifically as either a Black or Hispanic/Latnix transgender women and having completed study visit week 12 of the parent study when objective intra-erythrocytic intracellular PrEP drug levels were collected via dried blood spot (DBS) assay. 


\section{Data Collection}

From July 2018 through August 2019, eligible participants from the parent PrEP demonstration project were contacted by study staff, in the order they completed week 12 data collection, and were invited to participate in a qualitative interview about PrEP uptake and adherence. Participants who expressed interest in participating were provided informed consent and were enrolled in the sub-study. A total of 30 transgender women (16 Hispanic/Latinx, 10 Black, and 4 mixed race) participated in one-on-one, semi-structured interviews held in the private offices of either the Los Angeles LGBT Center or the Family Health Centers of San Diego. The interviews were offered in either English $(n=25)$ or Spanish $(n=5)$. The interviews lasted approximately one hour and were conducted by trained study staff who were members of the transgender women community and familiar to participants from their involvement in the parent demonstration project.

Interviewers followed a semi-structured qualitative interview guide developed by the study team in collaboration with community members and key stakeholders. As mentioned above, open-ended questions were developed in accordance with the guiding socioecological framework and based on hypothesized barriers to PrEP uptake and adherence identified in previous work with transgender women as well as the expert opinions of the research team and key community stakeholders. Questions asked about general attitudes, experiences, and beliefs about PrEP, as well as the individual-, interpersonal-, community-, and structurallevel barriers that were hypothesized to potentially influence PrEP uptake and adherence. After the interview, participants completed a brief socio-demographic survey and were compensated $\$ 50$ for their participation.

To gain additional insights into these barriers from the perspective of providers, a list of HCP known to be involved in the provision of PrEP to Black and Hispanic/Latinx transgender women (e.g., Linkage Program Coordinators, PrEP Navigators, Primary Care Providers) were identified from two Southern California-based LGBTQ-serving community health service organizations, with transgender-specific programming, and invited to participate in one-on-one interviews about their perceptions of the possible barriers to PrEP uptake and adherence among transgender women of color. An email explaining the interview and requesting participation was sent out to list of 13 providers and follow up emails, phone calls were made until 10 providers had participated in the interview (7 based in Los Angeles and 3 based in San Diego). No providers refused to participate however three did not respond to the email request.

Participant and provider interviews were audio recorded and then transcribed verbatim for data analysis. All study protocols were approved by the Institutional Review Boards of the RAND Corporation and the University of California, San Diego.

\section{Sociodemographic Surveys}

Participants were asked to complete a brief questionnaire collecting data on sociodemographics (age, race/ethnicity, gender identity, sexual identity, educational attainment, employment status, housing status, and relationship status) as well as mental health, recent substance use, and recent sexual risk behavior. Depressive symptoms were assessed using the Patient Health Questionnaire depression scale (PHQ-8) [23], a 8-item self-report scale used to screen for clinically significant depressive symptoms. The PHQ-8 is scored on a scale of $0-24$, with a score of $5,10,15$, and 20 considered as the cutpoints for mild, moderate, moderatesevere, and severe depression, respectively. Symptoms of generalized anxiety were assessed using the Generalized Anxiety Disorder Assessment (GAD-7) [24], a 7-item selfreport scale used to identity probable cases of anxiety disorder. The GAD-7 is scored on a scale of $0-21$, with a score of 5,10 , and 15 considered as the cutpoints for mild, moderate, and severe anxiety, respectively. Symptoms of PostTraumatic Stress Disorder (PTSD) were assessed using the Primary Care PTSD Screen (PC-PTSD) [25], a 4-item selfreport scale used to screen for PTSD. The scale had answers of "yes/no" and answering "yes" to any three or more items was considered a probable indication of PTSD. Substance use in previous 30 days was assessed by asking participants if they had engaged in the use of the following during the previous 30 days: binge drinking ( 5 or more drinks in a sitting), marijuana, poppers (alkyl nitrites), cocaine, methamphetamine, party drugs (e.g., MDMD, ecstasy, ketamine, GHB), or erectile dysfunction drugs (e.g., Cialis, Viagra). The questionnaire also asked participants if they had engaged in sex while high or drunk in the past 30 days as well as how long they had been taking PrEP.

A brief sociodemographic questionnaire was also administered to the HCP prior to interview in order to collect basic data on age, gender, education attainment, job title, length of time providing healthcare services to transgender women, and length of time providing PrEP-related healthcare services.

\section{Linked PrEP Adherence Data}

PrEP adherence was assessed in the parent demonstration project by collecting quantitative intracellular tenofovirdiphosphate (TFV-DP) levels in retrospect on batched, banked dried blood spot (DBS) samples starting in week12 of the parent demonstration project. Once analyzed, the DBS results were linked back to the sub-study participants through their assigned unique participant identification 
numbers. Adherence categories were defined based on recommended TFV-DP steady-state concentration levels of $1250 \mathrm{fmol} /$ punch or higher considered perfect adherence (7 doses/week), 700-1249 fmol/punch considered adherent/ protected (4-6 doses/week), and 699 or below fmol/punch or less considered non-adherent ( $<4$ doses/week) [26, 27].

\section{Data Analysis}

We conducted qualitative content analysis of the interview transcripts. Qualitative content analysis iteratively categorizes and reviews data to draw conclusions based on implicit and explicit meaning within the text [28]. All transcripts were uploaded to NVIVO 12 [29], a qualitative data analysis software program, for data organization and analysis. First, a member of the study team independently read $10 \%$ of the interviews and documented the preliminary emergent themes. Next, a draft codebook was developed from these preliminary emergent themes around barriers at each level of the socioecological framework. Themes were defined as codes or collections of codes with similar patterns of response. The codebook was shared with the study team, who also read $10 \%$ of the transcripts, provided feedback and made revisions until a codebook was further refined and finalized. Next, using the codebook, one team member coded all transcripts. New codes not contained in the codebook were added when at least three different participants mentioned the topic. After initial coding was completed, $20 \%$ of randomly selected transcripts were double coded by a second senior study member resulting in excellent interrater reliability (Cohen's Kappa $=0.84)$. Coding discrepancies were discussed until consensus was reached. In the event consensus wasn't reached, we consulted a third senior research team member and a decision was made based on their recommendation. We utilized the consolidated criteria for reporting qualitative research (COREQ) checklist to ensure systematic application of qualitative data analysis and presentation of our findings [30]. COREQ is a 32-point item checklist that ensures researchers report the pertinent components of qualitative studies including research team, study methodology, study findings, context of participants, and data interpretation [30].

\section{Results}

\section{Participant Characteristics}

Socio-demographic, PrEP adherence, mental health, and substance use data for the transgender women in this study are presented in Table 1.

The average age of the transgender women participants was 29.8 years [standard deviation $(\mathrm{SD})=8.6$ ]. More than
Table 1 Transgender women demographic characteristics $(\mathrm{N}=30)$

\begin{tabular}{|c|c|}
\hline & Mean (SD); N (\%) \\
\hline Age (years; range 21-47) & $29.8(8.6)$ \\
\hline \multicolumn{2}{|l|}{ Racial/ethnic identity } \\
\hline Hispanic/Latina/Latinx & $16(53.3)$ \\
\hline Black/African American & $10(33.3)$ \\
\hline Mixed Black and/or Hispanic/Latinx with other & $4(13.3)$ \\
\hline \multicolumn{2}{|l|}{ Interview language (preferred) } \\
\hline Spanish & $5(16.6)$ \\
\hline English & $25(83.3)$ \\
\hline \multicolumn{2}{|l|}{ Current gender identity (may select multiple) } \\
\hline Trans woman/trans female/trans feminine & $22(73.33)$ \\
\hline $\begin{array}{l}\text { Non-binary/gender queer/gender nonconform- } \\
\text { ing }\end{array}$ & $3(10.0)$ \\
\hline Female/woman & $8(26.6)$ \\
\hline \multicolumn{2}{|l|}{ Current sexual identity (may select multiple) } \\
\hline Straight/heterosexual & 14 (46.6) \\
\hline Bisexual & $3(10.0)$ \\
\hline Lesbian/same gender loving woman & $2(6.7)$ \\
\hline Gay/same gender loving man & $3(10.0)$ \\
\hline Pansexual & $5(16.6)$ \\
\hline Asexual & $1(3.3)$ \\
\hline Demisexual & $1(3.3)$ \\
\hline Other/queer/trans or nonconforming oriented & $4(13.3)$ \\
\hline \multicolumn{2}{|l|}{ Educational attainment } \\
\hline Less than high school & $2(6.7)$ \\
\hline High school diploma/GED & $9(30.0)$ \\
\hline Some college/Associate's degree & $11(36.7)$ \\
\hline 4-year college/Bachelor's degree & $6(20.0)$ \\
\hline Some graduate school/Graduate degree & $2(6.7)$ \\
\hline \multicolumn{2}{|l|}{ Legal employment (last 6 months) } \\
\hline Yes & $16(53.3)$ \\
\hline No & $14(46.7)$ \\
\hline \multicolumn{2}{|l|}{ Housing status } \\
\hline Stably housed & $23(76.7)$ \\
\hline Supportive/transitional housing & $3(10.0)$ \\
\hline Shelter & $2(6.7)$ \\
\hline Streets/squatting/abandoned building & $2(6.7)$ \\
\hline \multicolumn{2}{|l|}{ Relationship status } \\
\hline Single & $22(73.3)$ \\
\hline Married & $1(3.3)$ \\
\hline In a committed relationship but not married & $4(13.3)$ \\
\hline Open relationship/other partners & $3(10.0)$ \\
\hline \multicolumn{2}{|l|}{ Engaged in sex work (last 12 months) } \\
\hline Yes & $10(33.3)$ \\
\hline No & $20(66.7)$ \\
\hline \multicolumn{2}{|l|}{ PrEP adherence (12 week dried blood spot assay) } \\
\hline Perfect adherence & $14(46.7)$ \\
\hline Protective adherence & $5(16.7)$ \\
\hline Suboptimal adherence & $11(36.7)$ \\
\hline Seroconversion (later in study) & $1(3.3)$ \\
\hline Length of time on PrEP (months) & $8.9(10.7)$ \\
\hline
\end{tabular}


Table 1 (continued)

\begin{tabular}{|c|c|}
\hline & Mean (SD); N (\%) \\
\hline \multicolumn{2}{|l|}{ Mental health } \\
\hline $\begin{array}{l}\text { Depressive disorder symptoms (PHQ-8; last } \\
2 \text { weeks) }\end{array}$ & $5.8(5.9)$ \\
\hline Mild symptoms & $6(20.0)$ \\
\hline Moderate symptoms & $5(16.7)$ \\
\hline Moderate/severe to severe symptoms & $2(6.7)$ \\
\hline $\begin{array}{l}\text { Anxiety disorder symptoms (GAD-7; last } \\
2 \text { weeks) }\end{array}$ & $6.1(5.7)$ \\
\hline Mild symptoms & $7(23.3)$ \\
\hline Moderate symptoms & $3(10.0)$ \\
\hline Severe symptoms & $4(13.3)$ \\
\hline PTSD symptoms (PC-PTSD; last 30 days) & $2.3(1.5)$ \\
\hline Yes (3 or more symptoms) & $14(46.7)$ \\
\hline No (2 or less symptoms) & $16(53.3)$ \\
\hline \multicolumn{2}{|l|}{ Substance use (indicated use during last 30 days) } \\
\hline $\begin{array}{l}\text { Binge drinking ( } 5 \text { or more drinks in one sit- } \\
\text { ting) }\end{array}$ & $10(33.3)$ \\
\hline Marijuana & $17(56.7)$ \\
\hline Alkyl nitrites (poppers) & $5(16.7)$ \\
\hline Cocaine (crack or powder) & $3(10.0)$ \\
\hline Crystal methamphetamine & $2(6.7)$ \\
\hline $\begin{array}{l}\text { Party drugs (MDMA, Ecstacy, Special K, } \\
\text { GHB) }\end{array}$ & $2(6.7)$ \\
\hline Erectile dysfunction drugs & $3(10.0)$ \\
\hline Sex while high or drunk & $13(43.3)$ \\
\hline
\end{tabular}

half (60\%) identified as Hispanic/Latina/Latinx and more than a third (37\%) identified as Black/African American. Most participants (70\%) identified their gender identity as transgender women/transgender female/transgender feminine and almost half (43\%) reported their sexual identity as straight/heterosexual. The majority of participants (63\%) had an associate's degree or higher and 53\% reported being employed in the previous 6 months. A majority of participants reported having stable housing (77\%) and being single (73\%). A third (33\%) reported having engaged in sex work in the previous year.

\section{PrEP Adherence}

Results of TFV-DP DBS assay revealed 14 (46.7\%) had perfect adherence (7 doses/week), 5 (16.7\%) were adherent/protected (4-6 doses/week), and 11 (36.7\%) were non-adherent ( $<4$ doses/week). Among the Black/African American transgender women in this sample, $3(30 \%)$ had perfect adherence, 2 (20\%) were adherent/protected, and 5 (50\%) were non-adherent. Among Hispanic/Latina/Latinx transgender women, $8(50 \%)$ had perfect adherence, 3 (18.8\%) were adherent/protected, and 5 (31.3\%) were nonadherent. Among mixed race/ethnicity transgender women,
Table 2 Healthcare provider demographic characteristics $(\mathrm{N}=10)$

\begin{tabular}{ll}
\hline & Mean (SD); N (\%) \\
\hline Age (years; range 31-49) & $40.1(6.3)$ \\
Gender & \\
Female & $5(50.0)$ \\
Male & $4(40.0)$ \\
Transgender woman & $1(10.0)$ \\
Educational attainment & \\
Bachelor's degree & $2(20.0)$ \\
Master's degree & $2(20.0)$ \\
Doctoral degree & $6(60.0)$ \\
Job title & \\
Infectious disease physician & $3(30.0)$ \\
Nurse practitioner & $2(20.0)$ \\
Linkage to care supervisor & $1(10.0)$ \\
PrEP navigator & $1(10.0)$ \\
Internist physician & $1(10.0)$ \\
Clinical pharmacist & $1(10.0)$ \\
Health services director & $1(10.0)$ \\
Length of time providing healthcare services to & $87.9(65.6)$ \\
transgender women (months) & \\
Length of time providing PrEP-related healthcare & $54.3(33.5)$ \\
services (months) & \\
\hline
\end{tabular}

$3(75 \%)$ had perfect adherence, and $1(25 \%)$ were non-adherent. While $60 \%$ of the participants were in the 21-29 years old range, there were no significant differences in level of adherence to PrEP based on age in the current sample.

\section{Mental Health}

Just under a quarter (23\%) of the transgender women reported moderate to severe depressive symptoms and 23\% reported moderate to severe symptoms of anxiety. Almost half (47\%) of the transgender women reported meeting criteria for PTSD according to the PC-PTSD screening tool.

\section{Substance Use}

One third $(33 \%)$ of the transgender women in the current study reported binge drinking during the past 30 days. Over half $(57 \%)$ reported using marijuana and almost half (43\%) reported having had sex while high or drunk during the past 30 days.

\section{HCP Characteristics}

Descriptive characteristics of the HCP interviewed are presented in Table 2. The average age of the $\mathrm{HCP}(\mathrm{N}=10)$ was 40.1 years $(\mathrm{SD}=6.3)$. Most $(60.0 \%)$ had a doctoral degree and half identified as female. HCPs had provided healthcare 
services to transgender women for an average of 87.9 months $(\mathrm{SD}=65.6)$ and had provided PrEP-related healthcare services for an average of 54.3 months $(\mathrm{SD}=33.5)$.

\section{Qualitative Findings}

Emergent themes were categorized by individual, interpersonal, community, and structural level barriers to PrEP uptake and adherence (Fig. 1). Within each barrier type, we present perceptions from both transgender women and HCP.

\section{Individual-Level Factors}

Four individual-level barriers to PrEP uptake and adherence emerged from the interviews: cost concerns, mental health concerns, substance use, and PrEP side effects including FHT interaction.

Cost Concerns The majority of participants (both transgender women and HCP) cited concerns about PrEP-related costs as a major barrier to both uptake and adherence to PrEP. Those transgender women taking PrEP expressed that the primary reason they were currently on PrEP was due to participation in the PrEP demonstration project (which provided PrEP free-of charge) and that they would not be on PrEP if they had to pay out-of-pocket for PrEP, co-pays, or any of the related medical costs:

I'm here with you because you give me the medicine for free. (23 years old, Latinx, 4 months on PrEP, perfect adherence)

Transgender women expressed that co-pays and auxiliary health services costs (i.e., doctor visits and ongoing laboratory testing) and lack of health insurance coverage were barriers to PrEP uptake:

When I was working with the bank, my health insurance didn't cover me coming to this doctor. I waited a whole year, a whole year that I risked myself with having sex with people. I don't think anyone would want to go somewhere that they have to pay an exorbitant amount to get something [PrEP]. (34 years old, Latinx, 6 months on PrEP, perfect adherence)

Additionally, while all participants had access to PrEP free-of-charge, many raised concerns about having to discontinue PrEP due to cost-related issues in the future.

Later if I get taken out of the [demonstration] program, will I have to buy the PrEP medication? What would it cost? That is what influences me too. (23 years old, Latinx, 4 months on PrEP, perfect adherence)

Lastly, many transgender women described other competing financial responsibilities, especially related to housing, and FHT and those being higher in priority than PrEP:

I think it [PrEP] can be very prohibitively expensive for some people, especially if you don't have insurance. And then you're also thinking, 'I want genderaffirming surgeries and procedures and hormones.' And your money is gonna go to that before PrEP, I can guarantee you. (24 years old, Black \& Latinx, 3 years and 8 months on PrEP, non-adherent)

Most HCP echoed these sentiments and elaborated on how lack of financial resources affects the ability of transgender women to adopt and remain on PrEP:

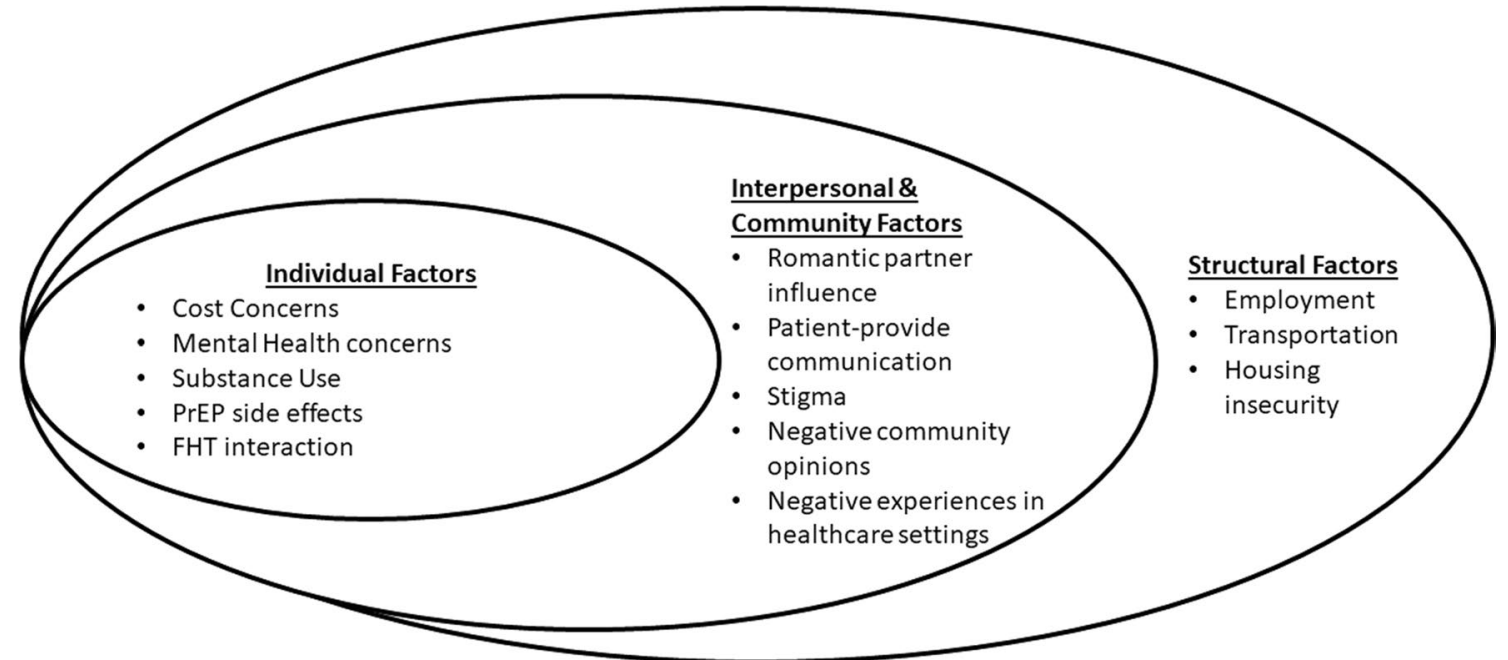

Fig. 1 Socioecological barriers to PrEP uptake and adherence among Black and Hispanic/Latinx transgender women in Southern California 
Transgender women are always looking for free ways of being able to get PrEP, but once a lot of those mechanisms run out after a certain amount of time, it's a complete impossibility to be able to afford to be on PrEP on their own, and to get the appropriate labs, and to be in care. (38 years old, Research Nurse Practitioner, 3 years and 3 months of healthcare service delivery to transgender women)

Mental Health Transgender women expressed that various mental health conditions (e.g., depression, anxiety, PTSD) either challenged their adherence to PrEP or motivated sustained adherence to PrEP. They described missing medication doses or completely stop taking PrEP due to the effects of dealing with mental health issues, which greatly affected their motivation to take PrEP and at times made PrEP less of a priority:

Sometimes, if you're not in the right state of mind, it can even add to that stress. Technically, you're just taking one pill a day. It seems like it's not a lot. But I know for me especially, I have PTSD, so if I'm triggered, and I'm super incredibly anxious, I can literally sit down and be anxious. Theoretically, I can take my PrEP medicine sitting down, but if my mind is everywhere, it's really difficult. (27 years old, Latinx, 9 months on PrEP, adherent/protected)

If you're depressed, you're not going to take anything. I'm not saying PrEP. Any pills. Because you feel depressed, you don't want to do anything. (28 years old, Black, 7 months on PrEP, non-adherent)

Similarly, several HCP described how dealing with mental health issues, particularly depression and anxiety, affected the ability for transgender women to start and remain adherent to PrEP and remain adherent to PrEP:

I have lots of depressed patients who say that they just can't take their antidepressants, their estrogen, or their PrEP. Their lack of motivation to even do that is a symptom of depression. So, self-care is a symptom of depression, and if PrEP is self-care, that's a barrier. (47 years old, Infectious Disease Physician, 18 years of healthcare service delivery to transgender women)

Conversely, some transgender women stated that they were able to stay adherent to PrEP despite dealing with mental health issues. For some, prioritizing their sexual health, amid a mental health crisis, was necessary and served as a coping mechanism:

I'm more concerned with being healthy and so that overrides every other concern. So, even if I'm depressed, I'm always going to put my health first.
(29 years old, Mixed Race, 1 year and 2 months on PrEP, perfect adherence)

Another participant described a realization of still being at risk for HIV, especially while dealing with depression, which served as the motivation to remaining adherent to PrEP:

Even when I feel depressed, I keep taking my pill [PrEP] every day because I know my depression will go away, but if I don't take my medicine, I'm going to be at risk to get HIV. (47 years old, Latinx, 8 months on PrEP, perfect adherence)

Substance Use Issues Several transgender women mentioned that their substance use behavior negatively affected their ability to stay adherent to PrEP. Substance use was usually described as occurring within social settings and with friends. Many described consuming alcohol and using other substances, which then led to forgetting to take PrEP:

There was one time that I was like out with friends. I'm at a friend's place, and it's like 7:00 a.m., and I'm like still a little drunk. And I'm like, "Shit. Like I forgot to bring PrEP. (30 years old, Latinx, 5 months on PrEP; perfect adherence at 12-week assessment, seroconverted later in the study)

Additionally, many transgender women described taking preemptive steps, prior to using substances, to ensure PrEP doses weren't missed:

Did I forget to take my PrEP that night? When I drank? Probably did. But I'm going out this weekend. I will make sure to take it before I go. (29 years old, Mixed Race, 1 year and 2 months on PrEP, perfect adherence)

A majority of HCP agreed that substance use problems affected the ability for transgender women to remain adherent to PrEP and shared stories of substance-using clients who struggled with PrEP adherence and missed PrEP-related medical appointments:

If they're on drugs, it's really hard, for them to show up for the appointment, or pick up their medications at the pharmacy, or come back and see their doctor every three months. So, there's going to be a lot of like no-shows for the appointments because you're on drugs. Getting PrEP would be the last thing on your list, because now you're addicted to drugs. (46 years old, Clinical Pharmacist, 9 years of healthcare service delivery to transgender women)

PrEP Side Effects and FHT Interaction Several transgender women cited concerns about negative interaction between PrEP and FHT as a major barrier to PrEP uptake and adher- 
ence. They expressed that several transgender women they knew worried that taking PrEP might reduce the effectiveness of FHT on their transitional process:

I've always had a thought like, does PrEP affect like my hormone? (23, Black/African American, 3 months on PrEP, perfect adherence)

HCP expressed that concerns about PrEP and FHT interaction was a major concern and barrier to PrEP uptake and adherence among transgender women:

They're more concerned about what PrEP does to their body, especially when they're on hormone therapy. They're a little bit concerned that there's going to be a drug-drug interaction with estradiol and Truvada, that Truvada might actually lower the potency of estradiol. (46 years old, Clinical Pharmacist, 9 years of healthcare service delivery to transgender women)

\section{Interpersonal-Level Factors}

Two interpersonal-level barriers to PrEP uptake and adherence emerged from the interviews: intimate/romantic partner influence and patient-provider communication.

Intimate/Romantic Partner Influence Several transgender women recounted how being in an intimate/romantic partnership influenced their ability to start PrEP and remain adherent. Many described that their partners were hesitant about the idea of them starting PrEP as it was perceived to indicate a lack of trust and loyalty within the relationship:

I remember when he found out [about PrEP] he asked, 'Why are you taking that blue pill?' I explained it to him and I remember he just had a really bad feeling. He just looked a certain way. (21 years old, Latinx, 3.5 months on PrEP, adherent/protected)

Similarly, several HCP discussed the influences of romantic partnerships on the uptake and adherence to PrEP among transgender women. They described how dishonesty within partnerships might cause one partner to steer the other away from PrEP and how interest in PrEP might be interpreted as cheating:

People have told me, 'well, my partner thinks that if I take PrEP, it means I'm cheating' and so there's a lot of blurred lines, or that the person doesn't trust their partner. (38 years old, Research Nurse Practitioner, 3 years and 3 months of healthcare service delivery to transgender women)

It could be that the partner may be having sex with other people as well and maybe not being honest. I always feel like those partners who are negative and who steer people away from PrEP are always kind of like hiding something because there's no reason why you shouldn't be on PrEP. (31 years old, PrEP Navigator, 2 years of healthcare service delivery to transgender women)

Patient-Provider Communication Transgender women described uncomfortable and sometimes negative experiences with HCP, who they perceived as culturally insensitive or lacking knowledge about transgender health, which served as barriers to PrEP uptake:

I told the doctor, I am transgender. I don't have ovaries, I don't have a uterus, I am not going to bleed, so stop asking me. And the doctor's like he's never heard or seen this. I am like, 'you're a doctor, man. You're looking at me like this? Like, come on.' And he went to a university, a big, fancy place. I don't get it. And I graduated from high school, I feel more informed about some things, this doctor should know more than me. (39 years old, Latinx, 2 years on PrEP, non-adherent)

[To get PrEP] you have to talk about sex with your doctor. A lot of providers nowadays, they don't even know how to interact with someone who's trans because they feel like they don't know what's appropriate to say or how to say things. They're just not very culturally sensitive or competent. (24 years old, Black/African American, 3 years and 8 months on PrEP, adherent/protected)

Several providers described hearing from patients about how negative experiences with their healthcare providerboth previous and current-inhibited their ability to start PrEP:

I hear a lot of people saying that their primary doctors don't understand trans health, and a lot of them reject the idea of PrEP, because the doctors themselves are uneducated, and stigmatize people. Particularly people who have used surgeons who aren't for a genderconfirming surgery, and asking their surgeons that they just write them a prescription for PrEP, and then being discriminated against blatantly. (44 years old, Director of Health Services \& Transgender Health Program, 15 years of healthcare service delivery to transgender women)

\section{Community-Level Factors}

Three community level barriers to PrEP uptake and adherence emerged from the transcripts: stigma, negative community opinions, and negative experiences in healthcare settings. 
Stigma Transgender women expressed that stigma related to being on PrEP and being seen seeking services in an HIV-related medical setting served as barriers to PrEP uptake and adherence. This included community sentiments of individuals on PrEP being perceived as overly sexually active, which contributed to the felt stigma described by participants:

I just think some of the reasons a transwoman would probably not want to be on it [PrEP], there's definitely backlash in the community with women wanting to take PrEP. It's frowned upon. I can't tell you how many times I've been called a whore or a slut. (21 years old, Latinx, 3.5 months on PrEP, adherent/ protected)

I feel like you come here and everyone's like, 'You have HIV.' I feel like that's the stigma here. If you're upstairs in the clinic, you're getting tested for something or even coming out of the room with a bandage, I'm embarrassed. I'm like, 'who's going to see me up there?' They're going to think that I'm getting blood pulled out because I'm a whore. (34 years old, Latinx, 6 months on PrEP, perfect adherence)

HCP agreed with transgender women and explained how PrEP related stigma remains a major barrier to PrEP uptake and adherence. HCP discussed the experience of stigma by transgender women in two ways: (1) PrEP being stigmatized within the transgender community and PrEP-using transgender women being perceived as promiscuous:

[There is] ignorance of what PrEP is. Some have a stigma about PrEP that's common just across society where they associate it with negative feelings about their own sexuality and what some would term 'promiscuity. (38 years old, Nurse Practitioner, 1.5 years of healthcare service delivery to transgender women)

And, (2) transgender women feeling stigmatized by HCP when they have conversations related to starting PrEP and staying on PrEP medications:

A lot of doctors don't know what PrEP is, and they find that you're on it, there's an automatic stigma that you're sexually promiscuous, and it's just disrespectful, and the person who's likely been disrespected in a lot of other ways just gets upset. If the provider starts asking about partner, once you start talking about partners, some providers may just be like, 'well, why can't you just have like one partner?' That's when transwomen of color say 'I'm not going to tell you anything, because you don't understand what I'm telling you and you're judging me (37 years old, Linkage to Care Supervisor, 8 years of healthcare service delivery to transgender women)
Negative Community Opinions Some HCP expressed that opinions, misinformation, medical mistrust, and myths about PrEP that are widely spread among the transgender women community influence uptake and adherence:

Word-of-mouth [communication] in the community can also steer them [transgender women] away, especially if you have that person who is just using condoms - and that's okay, too. But then it also creates the domino effect to other girls who are interested in PrEP, but they're just like, 'Oh, PrEP is not going to be right for me. There's a lot going on with PrEP.' A lot of times, they take these side effects that happen with PrEP and they kind of run with them a little bit, and that's what kind of scares transwomen away a little bit (31 years old, PrEP Navigator, 2 years of healthcare service delivery to transgender women)

There's a community who can be variably educated about medical issues. So word travels quickly. In a tighter-knit community, it doesn't take long for things to kind of get around. It's important that those be the sort of factual ideas, and not sort of the speculative or kind of fear-based ones. (39 years old, Infectious Disease Physician, 4.5 years of healthcare service delivery to transgender women)

There is mistrust of the medical community, because many have had negative experiences being misgendered in the clinical setting. But once you get past that barrier, I think in general, it's just like counselling anyone else about PrEP. (39 years old, Infectious Disease Physician, 4.5 years of healthcare service delivery to transgender women)

Negative Experiences in Healthcare Settings Transgender women described having negative experiences within healthcare settings, particularly related to being misgendered or treated in a disrespectful manner by healthcare staff, which dissuaded them from going to those spaces and impacts uptake and adherence to PrEP:

"When trans women go somewhere and they don't want to call your name, it's a pain in the ass. Like being misgendered or misnamed. If you don't feel comfortable there, you're not going to be able to feel good and you're not going to take it [PrEP]." (47 years old, Latinx, 8 months on PrEP, perfect adherence)

There was one time when I came in for a study and they were doing labs and I saw on their tubes that they put male. I was upset with them and talked to someone about why did they write this down here, because I have transitioned, I am female. (30 years old, Black/ African American, 3 years on PrEP, perfect adherence) 


\section{Structural-Level Factors}

We identified three structural level barriers to PrEP uptake and/or adherence: employment, reliable transportation, and housing insecurity/homelessness.

Employment Transgender women described demanding and sometimes irregular work schedules being a barrier to making PrEP-related medical appointments:

If you have a job, it can make scheduling difficult. Let's say you're working full time, you might not be able to make it here [hospital/clinic] in the time that the doctor is available to see me. (27 years old, Latinx, 9 months on PrEP, adherent/protected)

A few HCP explained how lack of stable employment served as a major barrier to PrEP uptake among transgender women as it relates to competing priorities and inconsistent insurance coverage:

If they're unemployed, PrEP would be the last thing on their list to get done. They would be more in search of getting a job, because they need to feed themselves and pay rent, if they have a place to stay. That could impact them not showing up for their appointments, or not picking up their medication. (46 years old, Clinical Pharmacist, 9 years of healthcare service delivery to transgender women)

As long as employment impacts your insurance coverage, that can impact ability to procure PrEP at regular intervals. If they're on a patient assistance program where it's not really paying for the drug that's the issue, the job and security might impact their ability to make it to appointments if they have to take whatever jobs are available. (39 years old, Infectious Disease Physician, 4.5 years of healthcare service delivery to transgender women)

Transportation Transgender women raised concerns about how lacking adequate means of transportation affected their ability to get to PrEP-related medical appointments. This included issues related to limited physical mobility, inconsistent bus schedule and unexpected public transportation delays:

My ex, and I have a hard time getting around. They have a lot of chronic pain which makes it hard to walk and stuff. Even when we were living together, we were living a mile and a half from [name of clinic] but even that was too much to walk. There was no direct bus route. It was a mile and a half, but it was $30 \mathrm{~min}$ on a bus. I would usually pick up PrEP for the both of us when I went. Yeah, it's a barrier.
(25 years old, Mixed Race, 4 years on PrEP, perfect adherence)

It was somewhat easier in the summer, but in the fall the trains take twice as long to get anywhere. I'm more panicked. And then, the buses in East LA are not as consistent as the ones in Central LA and West. (23 years old, Latinx, 9 months on PrEP, perfect adherence)

These sentiments were echoed by several HCP who described transportation constraints being a major barrier to adherence among transgender women due to feeling unsafe and uncomfortable in public transportation and not having enough resources to buy a car:

Transportation is a big thing because a lot of times, the transgender community, if they don't have any money or a car, they couldn't make it to their appointments and, therefore, you're not going to get a renewal prescription for your PrEP. And that could definitely be a hurdle. In addition to that, let's say if they were able to get transportation to get here - if they're 15 min late, their appointment might have to be rescheduled, and that's another burden for them. (46 years old, Clinical Pharmacist, 9 years of healthcare service delivery to transgender women)

Transportation can affect it, because they [transgender women] feel threatened, they don't feel safe, they need to present different genders to travel, they live far away, so I totally agree. Many of us had ideas to do PrEP, like telemedicine. (49 years old, Physician, 5 years of healthcare service delivery to transgender women)

Housing Insecurity/Homelessness Transgender women mentioned housing insecurity and experiencing homelessness as a major barrier to PrEP uptake and adherence. They elaborated that attaining stable housing was a higher priority than getting on PrEP:

For trans women who don't have stable housing, I can imagine PrEP isn't even on their list of priorities. (25 years old, Mixed Race, 4 years on PrEP, perfect adherence)

Additionally, for transgender women experiencing homelessness and/or living in shelters, many shared that their PrEP were sometimes stolen on the street or confiscated at shelters and medicine being administered during limited time windows, which made adherence difficult:

Because of my living situation, because I don't have a home, I find it hard to keep track of my medication. I've had my medication stolen from me, I've had my medication taken away from me, through the Depart- 
ment of Sanitation, they took my tent and my personal belongings, which had my medication. I went to the police to retrieve my stuff and my medication wasn't in there. So, I had to file a report and I had to wait till I got more medication to get back on it, and I was off of it [PrEP] for a week and a half. (39 years old, Latinx, 2 years on PrEP, non-adherent)

The shelter where I'm at now, they have a specific room for it[medication] because there's so many people and because there's so little staff - you can only have access to it at certain times. So, it makes it difficult for people that are trying to work or go to school, to where they can't always be there for that. It's never open when they're there. (23 years old, Latinx, 9 months on PrEP, perfect adherence)

HCP named homelessness and housing insecurity as one of the top issues facing the transgender women community in general, which directly affects their general health and wellbeing and ability to stay adherent to PrEP:

I have lots of trans patients and mostly transwomen who are homeless and will tell me that their medications get stolen a lot. So, just being able to have a safe place for them could be problematic. (38 years old, Infectious Disease Physician, 7 years of healthcare service delivery to transgender women)

\section{Discussion}

This study extends previous research that has examined the barriers to PrEP uptake among sexual minority communities to specifically address the barriers to PrEP uptake and adherence affecting a sample transgender women of color with varying PrEP adherence levels. We identified individual, interpersonal, community and structural barriers to PrEP uptake and adherence from the perspective of Black and Hispanic/Latinx transgender women and a sample of HCP in Southern California. While the majority of previous research on PrEP among transgender women has been related to knowledge, awareness and willingness to use PrEP [7, 13, $31,32]$, this research focuses on factors related to PrEP uptake and adherence among Black and Hispanic/Latinx transgender women with additional data on PrEP adherence levels . This work is paramount to informing ongoing efforts to reduce risk for HIV transmission and curb the heavy burden of HIV among Black and Hispanic/Latinx transgender women, a group disproportionately affected by HIV.

Overall, participants in our sample suggested that structural-level factors were a major barrier to PrEP uptake and adherence among transgender women. These included: lack of employment opportunities, lack of reliable transportation, and housing insecurity and/or homelessness. Our findings are consistent with other studies that have investigated structural barriers to PrEP uptake and adherence among transgender women [33, 34]. Addressing structural-level factors is paramount to maximizing the success of PrEP programs especially among Black and Hispanic/Latinx transgender women. A prototype for a possible intervention program is the A.S.K-PrEP program, a five-session peer navigation program that linked transgender women $(\mathrm{N}=58)$ in California with peer navigators who assessed PrEP eligibility and linked participants with ancillary support services such as mental health services, housing, substance abuse treatment, FHT, and food resources, based on individual needs [35]. Through this program, $90 \%$ of transgender women were linked to PrEP and at the 90-day follow-up point, $81 \%$ remained adherent to PrEP [35]. Given the considerable structural barriers to PrEP reported by transgender women, likely exacerbated by the coronavirus disease (COVID-19) pandemic, PrEP programs should explore how telemedicine and flexible medication delivery services might reduce the need for in-person visits and improve access to PrEP. Additionally, providing job training programs, resume workshops, job interview practice sessions, mentorship, and organizing job fairs with employers' keen on hiring transgender women might help reduce unemployment rates among transgender women, which has strong implications for PrEP uptake and adherence. Lastly, providing bus token or covering the cost of ride-sharing services might help to address the transportation barrier. Addressing these structural barriers is imperative to moving the needle as it relates to HIV prevention and reducing HIV transmission among Black and Hispanic/Latinx transgender women.

Other key barriers identified by the transgender women included the perception of PrEP-associated medical costs being high, mental health, and substance use problems that interfered with PrEP uptake and/or adherence, fear of PrEP and hormone interactions, negative prior experiences in healthcare settings, and medical mistrust. These findings are in line with previous studies that have found similar barriers to PrEP among transgender women $[33,36]$. While PrEP coverage by private insurance providers has increased, especially with the recent grade A designation by the U.S. Preventive Services Task Force [37], there remains an expressed perception that PrEP is unaffordable among some transgender women. It is important that campaigns aiming to increasing PrEP uptake among Black and Hispanic/Latinx transgender women highlight insurance coverage options and other available assistance programs within the jurisdiction (e.g. Gilead Copay Assistance Program and the recently instituted Ready, Set, PrEP Assistance Program (PrEP-AP) by the U.S. Department of Health and Human Services) that help defray personal costs of PrEP medications and the associated health services. It is also important to leverage existing culturally 
appropriate and gender-affirming community-level health interventions, by incorporating PrEP awareness programming, which might help increase PrEP uptake and adherence Black and Hispanic/Latinx transgender women.

The finding that mental health and substance use problems impacted PrEP uptake and adherence highlights the need to routinely screen Black and Hispanic/Latinx transgender women for these concerns during health care visits and provide referrals to mental health and substance use cessation services as needed. It is also important to explore concerns about possible FHT and PrEP interaction. A recent study among transgender women, found lower blood levels of PrEP with the presence of FHT could bolster fears of possible PrEP/FHT among transgender women, but the sample was relatively small $(\mathrm{N}=20)$, and importantly, the study found no changes in the hormone levels [38]. FHT is often a high priority for transgender women and a recent study found that $60 \%$ of transgender women not currently on PrEP would be more likely to take PrEP if provided at the same clinic that provided FHT [16]. Consequently, further studies are needed to rule out possible drug-drug interactions between FHT and PrEP among transgender women. Leveraging FHT visits to discuss PrEP and possibly bundling the provision of PrEP with FHT may be one way to increase access and capitalize on motivation among some transgender women to attend medical appointments for FHT. Lastly, medical mistrust and negative experiences in the healthcare setting were discussed by both transgender women and HCP. It is important to establish intentional partnerships between healthcare institutions and transgender women to have open and honest discourse about medical mistrust and legacies of racism and transphobia in the medical community [14].

Participants mentioned that interpersonal-level factors such as intimate/romantic partner influences, stigma, and negative community opinions about PrEP were also likely to impact both uptake and adherence to PrEP. Previous research has shown that stigma related to PrEP use, especially assumptions that PrEP is associated with engaging in risker sex practices is a major barrier to PrEP uptake among transgender women [10]. Breaking the stigma and dispelling the mistruths associated with PrEP is pivotal to increasing PrEP uptake among Black and Hispanic/Latinx transgender women. Participants also mentioned the importance of PrEP campaigns prominently featuring transgender women of color and also provide talking points to effectively negotiate PrEP use, especially within the context of intimate and romantic partnerships. This is particularly important because interest in PrEP has been demonstrated to be lower among transgender women who believe PrEP is for individuals who are promiscuous [39]. It is imperative that myths and conspiracy theories about PrEP are acknowledged and addressed by trusted key opinion leaders and major influencers within the transgender women community. An innovative approach may be to partner with popular Black and Latinx transgender social media influencers and media personalities (e.g., Ts Madison and Mj Rodriguez) to leverage their extensive social media following and respect within the community to dispel common PrEP myths and provide evidence-based sexual health information. Additionally, healthcare facilities that serve transgender women should institute policies that mandate sensitivity and cultural humility trainings for $\mathrm{HCP}$ and frontline staff involved in the provision of transgender healthcare services. Lastly, it is important that HCP consider how anti-Black racism (i.e. institutionalized police brutality and social injustice), transphobia (i.e., abuse and murder of transgender women of color), immigration status (i.e., threat of unlawful deportations), and other societal level policies may serve as barriers that prevent any transgender women from accessing quality healthcare services. HCP should actively work towards addressing these problems by creating brave spaces where PrEP and other healthcare services are offered that also actively affirms that that Black Lives Matter, Trans Lives Matter, and Immigrant Lives Matter.

Several limitations should be considered while interpreting the study findings. Given that our data were derived from a PrEP demonstration trial among relatively younger Black and Latinx/Hispanic transgender women in Southern California, these findings may not be generalizable to transgender women of other races, ethnicities, ages, or those in different geographical regions. Future studies should seek to better understand the relationships between factors at multiple levels and possible causal pathways between multilevel barriers to PrEP uptake and PrEP adherence for Black and Hispanic/Latinx transgender women. Additionally, the responses of participants may have been influenced by social desirability bias, especially since all interviews were conducted by transgender women. Despite these limitations, major strengths of the study include a focus on PrEP uptake and adherence among Black and Hispanic/Latinx transgender women who have access to PrEP as part of an ongoing demonstration project as opposed to simply PrEP knowledge and acceptability, including the perspectives of both transgender women and HCP, quantitative data on PrEP adherence among participants in our sample, and adaptation of the socioecological model [40] to the study design and data analysis.

To conclude, we identified barriers to PrEP uptake and adherence that fall along multiple socioecological levels from interviews conducted with Black and Hispanic/Latinx transgender women and HCP in Southern California. PrEP marketing and promotional materials should be developed after consultation with transgender women community members and stakeholders and should prominently feature and specifically target Black and Hispanic/Latinx transgender women to facilitate PrEP uptake and adherence among this group. Additionally, interventions that target structural 
level barriers to PrEP uptake among Black and Hispanic/ Latinx transgender women (e.g., employment, transportation, housing) should be prioritized and implemented in order to increase PrEP uptake and improve adherence.

Acknowledgements This project was funded by Grant R21DA044073 (PI: Storholm) from the National Institute on Drug Abuse (NIDA). Drs. Storholm and Ogunbajo were also supported by R03DA043402 (PI: Storholm). Drs. Ober, Bogart, Reback and Storholm acknowledge additional support from the National Institute of Mental Health (P30MH58107). Dr. Morris was funded by the parent study PrEP Linkage, Adherence \& Pharmacology in Transgender Persons (PR15-SD-021) from the California HIV/AIDS Research Program. The authors would like to thank the participants of the study as well as the study interviewers Chloe Opalo and Alvy Rangel without whom this study would not have been possible.

\section{Compliance with Ethical Standards}

Conflict of interest The authors have no relevant financial or non-financial interests to disclose and have no conflicts of interest to declare that are relevant to the content of this article.

\section{References}

1. Control CfD, Prevention. HIV and transgender people. CDC website. 2019.

2. Becasen JS, Denard CL, Mullins MM, Higa DH, Sipe TA. Estimating the prevalence of HIV and sexual behaviors among the US transgender population: a systematic review and meta-analysis, 2006-2017. Am J Public Health. 2019;109(1):e1-8.

3. Poteat T, Reisner SL, Radix A. HIV epidemics among transgender women. Curr Opin HIV AIDS. 2014;9(2):168-73.

4. Grant RM, Lama JR, Anderson PL, McMahan V, Liu AY, Vargas $\mathrm{L}$, et al. Preexposure chemoprophylaxis for HIV prevention in men who have sex with men. N Engl J Med. 2010;363(27):2587-99.

5. Deutsch MB, Glidden DV, Sevelius J, Keatley J, McMahan V, Guanira J, et al. HIV pre-exposure prophylaxis in transgender women: a subgroup analysis of the iPrEx trial. Lancet HIV. 2015;2(12):e512-9.

6. Donnell D, Baeten JM, Bumpus NN, Brantley J, Bangsberg DR, Haberer JE, et al. HIV protective efficacy and correlates of tenofovir blood concentrations in a clinical trial of PrEP for HIV prevention. J Acquir Immune Defic Syndr. 2014;66(3):340.

7. Hoagland B, De Boni RB, Moreira RI, Madruga JV, Kallas EG, Goulart SP, et al. Awareness and willingness to use pre-exposure prophylaxis (PrEP) among men who have sex with men and transgender women in Brazil. AIDS Behav. 2017;21(5):1278-87.

8. Draper BL, Oo ZM, Thein ZW, Aung PP, Veronese V, Ryan C, et al. Willingness to use HIV pre-exposure prophylaxis among gay men, other men who have sex with men and transgender women in Myanmar. J Int AIDS Soc. 2017;20(1):21885.

9. Jalil EM, Grinsztejn B, Velasque L, Makkeda AR, Luz PM, Moreira RI, et al. Awareness, willingness, and PrEP eligibility among transgender women in Rio de Janeiro, Brazil. J Acquir Immune Defic Syndr. 2018;79(4):445-52.

10. de Carvalho NP, Mendicino CCP, Cândido RCF, Alecrim DJD, de Pádua CAM. HIV pre-exposure prophylaxis (PrEP) awareness and acceptability among trans women: a review. AIDS Care. 2019;31:1-7.

11. Poteat T, Wirtz A, Malik M, Cooney E, Cannon C, Hardy WD, et al. A gap between willingness and uptake: Findings from mixed methods research on HIV prevention among black and latina transgender women. J Acquir Immune Defic Syndr. 2019;82(2):131-40.

12. Eaton LA, Matthews DD, Driffin DD, Bukowski L, Wilson PA, Stall RD, et al. A multi-US city assessment of awareness and uptake of pre-exposure prophylaxis (PrEP) for HIV prevention among black men and transgender women who have sex with men. Prev Sci. 2017;18(5):505-16.

13. Restar AJ, Kuhns L, Reisner SL, Ogunbajo A, Garofalo R, Mimiaga MJ. Acceptability of antiretroviral pre-exposure prophylaxis from a cohort of sexually experienced young transgender women in two US cities. AIDS Behav. 2018;22(11):3649-57.

14. Sevelius JM, Keatley J, Calma N, Arnold E. 'I am not a man': trans-specific barriers and facilitators to PrEP acceptability among transgender women. Global Public Health. 2016;11(7-8):1060-75.

15. Wang Z, Lau JT, Yang X, Cai Y, Gross DL, Ma T, et al. Acceptability of daily use of free oral pre-exposure prophylaxis (PrEP) among transgender women sex workers in Shenyang, China. AIDS Behav. 2017;21(12):3287-98.

16. Hood JE, Eljallad T, Abad J, Connolly M, Heumann C, Fritz J, et al. Getting pre-exposure prophylaxis to high-risk transgender women: lessons from Detroit, USA. Sexual Health. 2018;15(6):562-9.

17. Rael CT, Martinez M, Giguere R, Bockting W, MacCrate C, Mellman W, et al. Barriers and facilitators to oral PrEP use among transgender women in New York City. AIDS Behav. 2018;22(11):3627-36.

18. Arrington-Sanders R, Hailey-Fair K, Wirtz AL, Morgan A, Brooks D, Castillo M, et al. Role of structural marginalization, HIV stigma, and mistrust on HIV prevention and treatment among young black latinx men who have sex with men and transgender women: perspectives from youth service providers. AIDS Patient Care STDs. 2020;34(1):7-15.

19. Bronfenbrenner U. Ecological models of human development. Read Dev Child. 1994;2(1):37-43.

20. Philbin MM, Parker CM, Parker RG, Wilson PA, Garcia J, Hirsch JS. The promise of pre-exposure prophylaxis for black men who have sex with men: an ecological approach to attitudes, beliefs, and barriers. AIDS Patient Care STDs. 2016;30(6):282-90.

21. Biello KB, Oldenburg CE, Mitty JA, Closson EF, Mayer KH, Safren SA, et al. The "safe sex" conundrum: anticipated stigma from sexual partners as a barrier to PrEP use among substance using MSM engaging in transactional sex. AIDS Behav. 2017;21(1):300-6.

22. Li J, Berg CJ, Kramer MR, Haardörfer R, Zlotorzynska M, Sanchez TH. An integrated examination of county-and individuallevel factors in relation to HIV pre-exposure prophylaxis awareness, willingness to use, and uptake among men who have sex with men in the US. AIDS Behav. 2019;23(7):1721-36.

23. Kroenke K, Strine TW, Spitzer RL, Williams JB, Berry JT, Mokdad AH. The PHQ-8 as a measure of current depression in the general population. J Affect Disord. 2009;114(1):163-73.

24. Spitzer RL, Kroenke K, Williams JB, Löwe B. A brief measure for assessing generalized anxiety disorder: the GAD-7. Arch Intern Med. 2006;166(10):1092-7.

25. Cameron RP, Gusman D. The primary care PTSD screen (PCPTSD): development and operating characteristics. Primary Care Psychiatry. 2003;9(1):9-14.

26. Landovitz RJ, Beymer M, Kofron R, Amico KR, Psaros C, Bushman L, et al. Plasma tenofovir-levels to support adherence to TDF/FTC pre-exposure prophylaxis for HIV prevention in MSM in Los Angeles, California. J Acquir Immune Defic Syndr. 2017;76(5):501.

27. Anderson PL, Liu AY, Castillo-Mancilla JR, Gardner EM, Seifert SM, McHugh C, et al. Intracellular tenofovir-diphosphate and 
emtricitabine-triphosphate in dried blood spots following directly observed therapy. Antimicrob Agents Chemother. 2018. https:// doi.org/10.1128/AAC.01710-17.

28. Mayring P. Qualitative content analysis. Compan Qual Res. 2004;1:159-76.

29. Edhlund B, McDougall A. Nvivo 12 essentials: Lulu.com; 2019.

30. Tong A, Sainsbury P, Craig J. Consolidated criteria for reporting qualitative research (COREQ): a 32-item checklist for interviews and focus groups. Int J Qual Health Care. 2007;19(6):349-57.

31. Garnett M, Hirsch-Moverman Y, Franks J, Hayes-Larson E, El-Sadr WM, Mannheimer S. Limited awareness of preexposure prophylaxis among black men who have sex with men and transgender women in New York city. AIDS Care. 2018;30(1):9-17.

32. Wilson E, Chen Y-H, Pomart WA, Arayasirikul S. Awareness, interest, and HIV pre-exposure prophylaxis candidacy among young transwomen. AIDS Patient Care STDs. 2016;30(4):147-50.

33. Cahill SR, Keatley J, Wade Taylor S, Sevelius J, Elsesser SA, Geffen SR, et al. "Some of us, we don't know where we're going to be tomorrow". Contextual factors affecting PrEP use and adherence among a diverse sample of transgender women in San Francisco. AIDS Care. 2019;32:1-9.

34. Brookfield S, Dean J, Forrest C, Jones J, Fitzgerald L. Barriers to accessing sexual health services for transgender and male sex workers: a systematic qualitative meta-summary. AIDS Behav. 2020;24(3):682-96.

35. Reback CJ, Clark KA, Rünger D, Fehrenbacher AE. A promising PrEP navigation intervention for transgender women and men who have sex with men experiencing multiple syndemic health disparities. J Community Health. 2019;44(6):1193-203.

36. Dankerlui D, Connolly M, Joseph C, Eljallad T, Dodard-Friedman I. P262 PrEP utilization among young transgender women, transgender men, and MSM in an urban community-based setting. BMJ; 2019.

37. Scott H, Volberding PA. HIV screening and preexposure prophylaxis guidelines: following the evidence. JAMA. 2019;321(22):2172-4.

38. Hiransuthikul A, Janamnuaysook R, Himmad K, Kerr SJ, Thammajaruk N, Pankam T, et al. Drug-drug interactions between feminizing hormone therapy and pre-exposure prophylaxis among transgender women: the iFACT study. J Int AIDS Soc. 2019;22(7):e25338.

39. Eaton LA, Kalichman SC, Price D, Finneran S, Allen A, Maksut J. Stigma and conspiracy beliefs related to pre-exposure prophylaxis (PrEP) and interest in using PrEP among black and white men and transgender women who have sex with men. AIDS Behav. 2017;21(5):1236-46.

40. McLeroy K, Steckler A, Bibeau D. The social ecology of health promotion interventions. Health Educ Q. 1988;15(4):351-77.

Publisher's Note Springer Nature remains neutral with regard to jurisdictional claims in published maps and institutional affiliations. 